\title{
Efeitos da correção cirúrgica de estenose mitral sobre o ritmo cardíaco
}

\author{
Carlos Augusto SCHMIDLIN*, Danton R. da Rocha LOURES*, Roberto Gomes de CARVALHO*, \\ Leonardo Andrade MULINARI*, Arleto Zacarias SILVA Jr.*, Maricélia BROMMELSTRÖET*, \\ Ricardo José CHOMA*, Sérgio SHIBATA*, Luciano Augusto LEITÃO*, Fábio Rodrigues SILVA*, \\ Frederico Thomaz ULTRAMARI*
}

RBCCV 44205-539

Schmidlin C A, Loures D R R, Carvalho R G, Mulinari L A, Silva Jr. A Z, Brommelströet M, Choma R J, Shibata S, Leitão L A, Silva F R, Ultramari F T - Efeitos da correção cirúrgica de estenose mitral sobre o ritmo cardíaco. Rev Bras Cir Cardiovasc 2001; 16(2): 128-35.

RESUMO: Objetivo: Determinar a freqüência de reversão de fibrilação atrial (FA) ao ritmo sinusal (SA) em pacientes com estenose mitral (EM) submetidos a tratamento cirúrgico e identificar prováveis fatores favoráveis ou desfavoráveis a este evento.

Casuística e Métodos: Estudo de caso-controle, envolvendo 53 pacientes com EM, sem acometimento de outras valvas, submetidos a correção cirúrgica. A população estudada apresentou as seguintes características: mulheres: 71,7\%; idade média: 42,4 anos; classe funcional III: 67,9\%; área mitral média: 0,92 $\mathrm{cm}^{2}$; átrio esquerdo médio: $56,0 \mathrm{~mm}$; ritmo antes da operação: SA: $51,0 \%$ e FA: $49,0 \%$. Os pacientes foram divididos em dois grupos de acordo com o ritmo apresentado no período pós-operatório tardio: grupo I, formado pelos pacientes que, após a operação, apresentavam ritmo SA e grupo II, constituído por aqueles que, no pós-operatório, estavam em FA.

Resultados: Dez (18,9\%) pacientes (em relação ao total; $38,5 \%$ em relação àqueles com $F A$ ) apresentavam FA no período pré-operatório e sofreram reversão para o ritmo SA no pós-operatório e em 2 pacientes (3,8\% em relação ao total; 7,4\% em relação àqueles em ritmo $S A$ ) houve degeneração do ritmo SA para FA. Houve diferença estatisticamente significativa $(p<0,05)$ entre os dois grupos apenas em relação a variável idade $(p=0,0456)$.

Conclusões: $A$ operação de correção de EM apresenta resultados insatisfatórios em relação à reversão da FA para ritmo SA, sugerindo a necessidade de associação de outro procedimento cirúrgico para restaurar o ritmo normal. Vários estudos tentaram identificar os fatores predisponentes à permanência e ao desenvolvimento de FA após a operação, porém foram obtidos resultados contraditórios. No presente estudo, a única variável que apresentou associação com a FA foi a idade avançada.

DESCRITORES: Estenose da valva mitral, cirurgia. Fibrilação atrial, fisiopatologia. Freqüência cardíaca, fisiologia.

\footnotetext{
Trabalho realizado na Disciplina de Cirurgia Torácica e Cardiovascular do Departamento de Cirurgia do Setor de Ciências da Saúde da Universidade Federal do Paraná (UFPR). Curitiba, PR, Brasil. Recebido para publicação em setembro de 2000.

* Da Disciplina de Cirurgia Torácica e Cardiovascular.

Endereço para correspondência: Carlos A. Schmidlin. Rua Tomozina, 395. Curitiba, PR, Brasil. CEP 80540-160. Tel. (41) 253-5311/343-3958. e-mail:danisacamoto@net.par.com.br
} 
Schmidlin C A, Loures D R R, Carvalho R G, Mulinari L A, Silva Jr. A Z, Brommelströet M, Choma R J, Shibata S, Leitão L A, Silva F R, Ultramari F T - Efeitos da correção cirúrgica de estenose mitral sobre o ritmo cardíaco. Rev Bras Cir Cardiovasc 2001; 16(2): 128-35.

\section{INTRODUÇÃO}

A prevalência de fibrilação atrial (FA) na população adulta é $0,4 \%$, mas sua freqüência aumenta com a idade para aproximadamente $2 \%$ a $4 \%$ nos indivíduos com 60 ou mais anos (1). Nos países ocidentais desenvolvidos, a cardiopatia reumática era a causa mais comum dessa arritmia. Atualmente, houve um declínio na freqüência de ambas cardiopatia reumática e FA conseqüente. A cardiopatia hipertensiva e a insuficiência cardíaca congestiva assumiram posição de destaque como anormalidades subjacentes associadas à FA crônica (2). Em nosso meio, no entanto, a cardiopatia reumática permanece como uma importante causa de FA crônica e suas complicações, como tromboembolismo sistêmico e cerebral, são freqüentemente observadas na prática clínica diária. Na verdade, essa arritmia representa o principal fator de risco para o desenvolvimento de eventos tromboembólicos (3).

$\mathrm{Na}$ estenose mitral (EM) pura, a FA crônica é uma das arritmias mais freqüentes ${ }^{(4-7)}$ sendo detectada em até $40 \%$ a $60 \%$ dos pacientes $(8,9)$. Muitos fatores foram propostos como contribuintes da patogenia da FA na EM tais como idade avançada e duração da valvopatia mitral reumática ${ }^{(5,6)}$, área da valva mitral(10) dilatação e fibrose do miocárdio atrial (11).

O reparo cirúrgico da obstrução mitral tem sido indicado para restaurar o ritmo sinusal (SA) e reduzir o risco de eventos tromboembólicos, além de corrigir a disfunção valvar. A identificação de fatores de risco independentes é importante para o desenvolvimento de estratégias de prevenção e tratamento dessa arritmia em pacientes com estenose mitral candidatos ao tratamento cirúrgico. $O$ objetivo do presente estudo foi determinar a freqüência de reversão de $F A$ ao ritmo sinusal em pacientes com EM e identificar prováveis fatores favoráveis ou desfavoráveis a este evento.

\section{CASUÍSTICA E MÉTODOS}

Foi realizado um estudo observacional longitudinal de caso-controle por análise de prontuários, envolvendo 53 pacientes submetidos a correção cirúrgica de EM pelo Serviço de Cirurgia Torácica e Cardiovascular do Hospital de Clínicas da Universidade Federal do Paraná, de janeiro de 1995 a dezembro de 1999.

Os pacientes constituintes da população estudada obedeciam aos seguintes critérios: 1) portadores de EM pura ou predominante sem acometimento de outras valvas, submetidos a correção cirúrgica, 2) realizados exames clínico, eletro- e ecocardiográfico (além de cateterismo cardíaco naqueles com idade igual ou superior a 40 anos) no período pré-operatório e, pelo menos, clínico e eletrocardiográfico no período pós-operatório.

A análise estatística foi realizada no Epi Info versão 6.04. Foram estudadas as seguintes variáveis: sexo, idade, número de operações da valva mitral, classe funcional pré-operatória, variáveis ecocardiográficas pré- e pós-operatórias (área da valva mitral, estenose mitral pura ou predominante, calcificação da valva mitral, tamanho do átrio esquerdo, gradiente transmitral médio, pressão sistólica da artéria pulmonar, fração de ejeção, septo interventricular), tipo de operação, ritmos pré- e pós-operatórios e mortalidade.

Os valores de referência para as variáveis analisadas estão na Tabela 1 e as características básicas da população estudada estão resumidas na Tabela 2.

O ritmo cardíaco foi monitorizado continuamente por pelo menos 3 dias após a operação e eletrocardiogramas foram obtidos diariamente por pelo menos 5 dias. Os pacientes foram acompanhados, em média, por um período de 20 meses, através de anamnese e exames físico, eletro e ecocardiogramas periódicos. Apenas 9 pacientes não foram submetidos a exame ecocardiográfico após a operação, em 14 foi realizado um ecocardiograma e, nos 30 restantes, dois ou mais. $O$ primeiro ecocardiograma foi realizado próximo ao sexto mês pós-operatório e o segundo, 2 anos após a operação.

Os pacientes foram divididos em dois grupos de acordo com o ritmo apresentado no acompanhamento pós-operatório tardio comprovado por eletrocardiogramas, desde que mantido por pelo menos 6 meses. O primeiro (grupo I) foi formado pelos pacientes que estavam em ritmo SA ou FA e, após a operação, permaneceram ou reverteram para o ritmo SA, respectivamente. O grupo II representou aqueles que estavam em ritmo SA ou FA e, no pósoperatório, adquiriram ou permaneceram em FA, respectivamente.

\section{RESULTADOS}

A evolução dos ritmos apresentados pelos pacientes pode ser observada na Figura 1. Dos 26 $(49,0 \%)$ pacientes que estavam em FA antes da operação, $16(61,5 \%)$ permaneceram em tal ritmo no período pós-operatório tardio, enquanto 10 deles $(38,5 \%)$ sofreram reversão para o ritmo SA. Vinte e sete $(51,0 \%)$ pacientes apresentavam-se em ritmo 
Schmidlin C A, Loures D R R, Carvalho R G, Mulinari L A, Silva Jr. A Z, Brommelströet M, Choma R J, Shibata S, Leitão L A, Silva F R, Ultramari F T - Efeitos da correção cirúrgica de estenose mitral sobre o ritmo cardíaco. Rev Bras Cir Cardiovasc 2001; 16(2): 128-35.

TABELA 1

VALORES DE REFERÊNCIA

\begin{tabular}{|c|c|c|}
\hline Faixa etária (anos) & $\begin{array}{l}1^{a}: 15-25 \\
2^{a}: 25-35 \\
3^{a}: 35-45 \\
4^{a}: 45-55\end{array}$ & $\begin{array}{l}5^{a}: 55-65 \\
6^{a}: 65-75 \\
7^{a}: 75-65\end{array}$ \\
\hline Grau de estenose mitral $\left(\mathrm{cm}^{2}\right)^{*}$ & \multicolumn{2}{|c|}{$\begin{array}{c}\text { Severa: } \leq 1 \\
\text { Moderada: } 1-2 \\
\text { Leve: }>2\end{array}$} \\
\hline Átrio esquerdo $(\mathrm{mm})^{\star \star}$ & \multicolumn{2}{|c|}{$\begin{array}{c}\text { Severamente aumentado: }>60 \\
\text { Aumentado: } 40-60 \\
\text { Normal: } \leq 40\end{array}$} \\
\hline Aumento da pressão sistólica da artéria pulmonar $(\mathrm{mmHg})^{\star \star \star}$ & \multicolumn{2}{|c|}{$\begin{array}{c}\text { Severo: }>60 \\
\text { Moderado: } 30-60 \\
\text { Normal: } \leq 30\end{array}$} \\
\hline Fração de ejeção (\%) ${ }^{* * *}$ & \multicolumn{2}{|c|}{$\begin{array}{c}\text { Reduzida: }<64 \\
\text { Normal } \geq 64\end{array}$} \\
\hline Septo interventricular $(\mathrm{mm})^{\star * *}$ & \multicolumn{2}{|c|}{$\begin{array}{c}\text { Aumentado: } \geq 12 \\
\text { Normal: }<12\end{array}$} \\
\hline
\end{tabular}

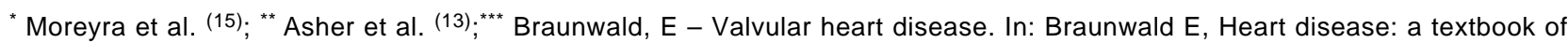
cardiovascular medicine. 5. ed. Philadelphia: W. B. Saunders, 1997: 1009.

TABELA 2

CARACTERÍSTICAS BÁSICAS DA POPULAÇÃO ESTUDADA NO PERÍODO PRÉ-OPERATÓRIO

\begin{tabular}{|c|c|}
\hline Sexo $(\%)$ & $\begin{array}{l}\text { Feminino: } 38(71,7) \\
\text { Masculino: } 15(28,3)\end{array}$ \\
\hline Idade média em anos & $42,4 \pm 12,8$ (16 a 81 anos) \\
\hline Número de operações da valva mitral (\%) & $\begin{array}{c}\text { Uma: } 30(56.6) \\
\text { Duas: } 20(37,7) \\
\text { Três: } 3(5,7)\end{array}$ \\
\hline Classe funcional pré-operatória (\%) & $\begin{array}{l}\text { I: } 2(3,8) \\
\text { II: } 12(22,6) \\
\text { III: } 36(67,9) \\
\text { IV: } 3(5,7)\end{array}$ \\
\hline Área mitral média $\left(\mathrm{cm}^{2}\right)$ & $0,92 \pm 0,25$ \\
\hline Grau de estenose mitral (\%) & $\begin{array}{c}\text { Severa: } 38(71,7) \\
\text { Moderada: } 15(28,3)\end{array}$ \\
\hline Estenose mitral pura ou predominante (\%) & $\begin{array}{c}\text { Pura: } 21(39,6) \\
\text { Predominante: } 32(60,4)\end{array}$ \\
\hline Calcificação da valva mitral (\%) & $\begin{array}{l}\text { Sim: } 21(39,6) \\
\text { Não: } 32(60,4)\end{array}$ \\
\hline Átrio esquerdo médio (mm) & $56,0 \pm 10,3$ \\
\hline Gradiente transmitral médio (mmHg) & $11,9 \pm 5,0$ \\
\hline Pressão sistólica da artéria pulmonar média (mmHg) & $61,6 \pm 21,8$ \\
\hline Fração de ejeção média em \% & $60,6 \pm 11,4$ \\
\hline Septo interventricular médio (mm) & $9,5 \pm 1,8$ \\
\hline Tipo de operação (\%) & $\begin{array}{c}\text { Comissurotomia: } 13(24,5) \\
\text { Comissurotomia e valvoplastia: } 5(9,4) \\
\text { Prótese biológica: } 22(41,5) \\
\text { Prótese metálica: } 13(24,5)\end{array}$ \\
\hline
\end{tabular}


Schmidlin C A, Loures D R R, Carvalho R G, Mulinari L A, Silva Jr. A Z, Brommelströet M, Choma R J, Shibata S, Leitão L A, Silva F R, Ultramari F T - Efeitos da correção cirúrgica de estenose mitral sobre o ritmo cardíaco. Rev Bras Cir Cardiovasc 2001; 16(2): 128-35.

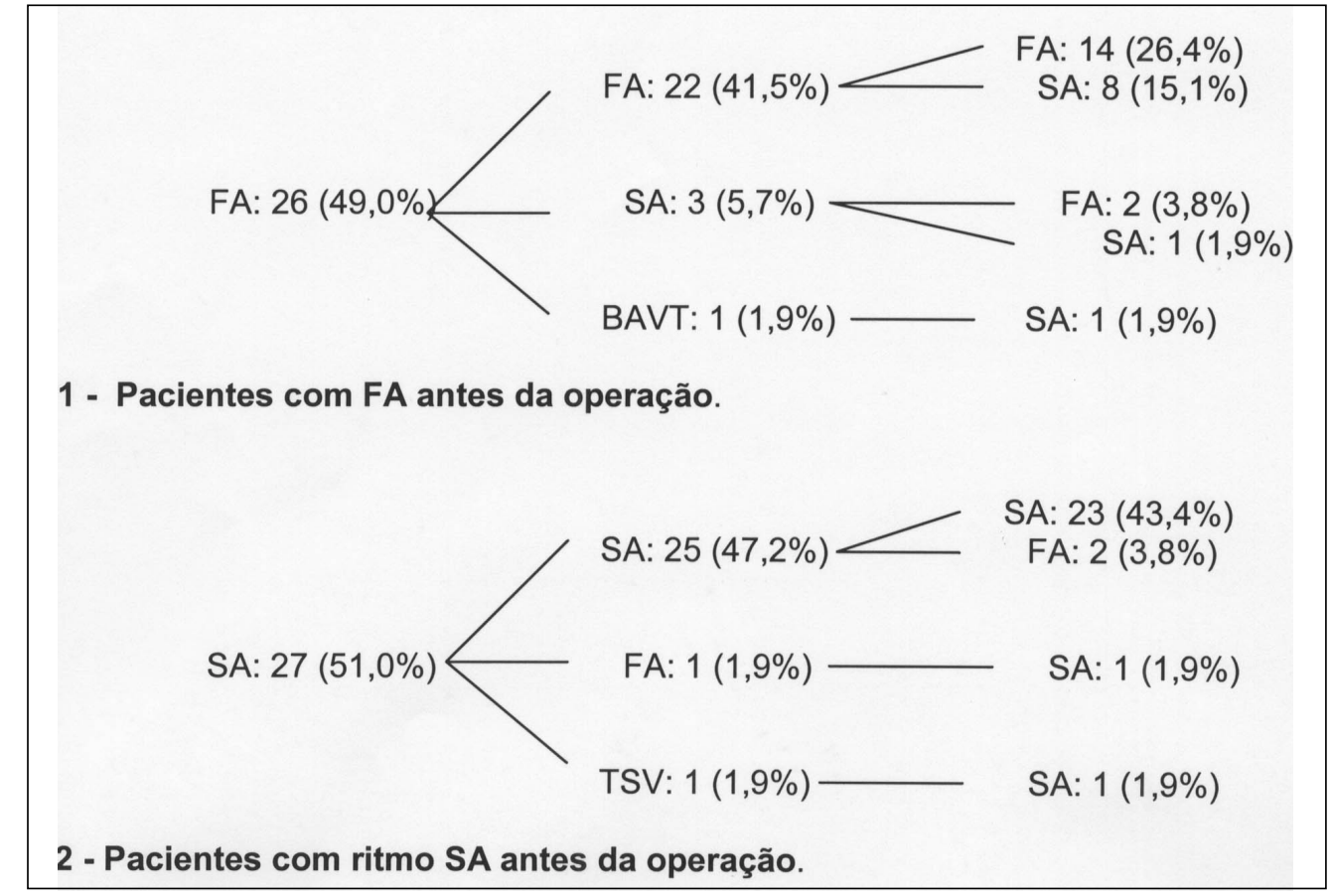

Fig. 1 - Evolução dos ritmos apresentados pelos pacientes. Acima (1), evolução dos pacientes com fibrilação atrial no período pré-operatório e abaixo (2), evolução dos pacientes com ritmo sinusal no período pré-operatório. Em ambos, o ritmo da coluna da esquerda é o pré-operatório, na coluna do meio está o ritmo pós-operatório intra-hospitalar e na coluna da direita, o ritmo pós-operatório tardio. FA: fibrilação atrial, SA: ritmo sinusal, BAVT: bloqueio atrioventricular total, TSV: taquicardia supraventricular.

SA no período pré-operatório, dos quais 25 (92,6\%) mantiveram-se com ritmo normal no pós-operatório tardio e apenas 2 deles $(7,4 \%)$ sofreram degeneração para FA.

Quanto ao início da arritmia nos pacientes com FA, não foi possível estabelecê-lo com exatidão. Tal dado pode ser observado na Tabela 3. Dos 26 pacientes que apresentavam FA antes da operação, 7 $(26,9 \%)$ possuíam apenas os eletrocardiogramas realizados no período pré-operatório. Quanto aos indivíduos que sofreram reversão da FA para ritmo SA, $4(40 \%)$ já apresentavam a arritmia há pelo menos 1 ano ou mais, $3(30 \%)$ há menos de 1 ano e em 3 (30\%) a duração da FA não pôde ser estimada.

Dos 53 pacientes, $35(66,0 \%)$ foram incluídos no grupo I. Desses, 25 (47,2\%) estavam em ritmo SA no período pré-operatório e permaneceram em tal ritmo após a operação e $10(18,9 \%)$ apresentavam FA no período pré-operatório e sofreram reversão para o ritmo normal no período pós-operatório tardio. O grupo II compreendeu $18(34,0 \%)$ pacientes, dos quais $16(30,2 \%)$ permaneceram em FA após a operação e em $2(3,8 \%)$ pacientes houve degeneração do ritmo SA para FA após a correção cirúrgica da EM.
Nos 10 casos em que houve mudança do ritmo cardíaco de FA para ritmo SA, 1 (10\%) paciente apresentou a reversão no período pós-operatório intra-hospitalar; os 9 restantes $(90 \%)$, no pós-operatório tardio. Os dois pacientes que sofreram degeneração do ritmo SA para FA, apresentaram tal evento no período pós-operatório tardio.

A Tabela 4 compara as diversas variáveis entre ambos grupos.

Houve diferença estatisticamente significativa $(p<0,05)$ entre os dois grupos apenas em relação a variável idade, tendo sido obtido $p=0,0456$. Quanto às demais variáveis foram obtidos os respectivos valores de $\mathrm{p}$ : número de operações da valva mitral: $p=0,1491$; classe funcional pré-operatória: $p=0,1953$; grau de estenose mitral: $p=0,2198$; estenose mitral pura ou predominante: $p=0,2061$; calcificação da valva mitral: $p=0,0890$; átrio esquerdo: $p=0,4460$; gradiente transmitral médio: $p=0,7066$; pressão sistólica da artéria pulmonar: $p=0,3950$; fração de ejeção: $p=0,7812$; sept $\mathrm{p}=0,4796$.

Dos pacientes com menos de 35 anos, 6,3\% apresentavam FA no período de acompanhamento 
Schmidlin C A, Loures D R R, Carvalho R G, Mulinari L A, Silva Jr. A Z, Brommelströet M, Choma R J, Shibata S, Leitão L A, Silva F R, Ultramari F T - Efeitos da correção cirúrgica de estenose mitral sobre o ritmo cardíaco. Rev Bras Cir Cardiovasc 2001; 16(2): 128-35.

TABELA 3

TEMPO DECORRIDO ENTRE O PRIMEIRO ELETROCARDIOGRAMA DEMONSTRANDO FA E A OPERAÇÃO DE CORREÇÃO DA EM

\begin{tabular}{|c|c|c|}
\hline PACIENTE & TEMPO & EVOLUÇÃO DO RITMO ** \\
\hline 1 & 13 anos & FA-FA-FA \\
\hline 2 & 11 anos & FA-FA-FA \\
\hline 3 & 10 anos & FA-FA-FA \\
\hline 4 & 6 anos & FA-FA-SA \\
\hline 5 & 5 anos & FA-SA-FA \\
\hline 6 & 4 anos e 4 meses & FA-FA-FA \\
\hline 7 & 2 anos e 9 meses & FA-FA-FA \\
\hline 8 & 2 anos e 4 meses & FA-FA-FA \\
\hline 9 & 1 ano e 8 meses & FA-FA-SA \\
\hline 10 & 1 ano e 5 meses & FA-FA-FA \\
\hline 11 & 1 ano e 3 meses & FA-SA-SA \\
\hline 12 & 1 ano & FA-FA-SA \\
\hline 13 & 8 meses & FA-FA-FA \\
\hline 14 & 4 meses & FA-FA-SA \\
\hline 15 & 3 meses & FA-FA-SA \\
\hline 16 & 2 meses & FA-FA-FA \\
\hline 17 & 1 mês & FA-FA-FA \\
\hline 18 & 1 mês & FA-FA-FA \\
\hline 19 & 1 mês & FA-BAVT-SA \\
\hline 20 & Pré-operatório * & FA-FA-FA \\
\hline 21 & Pré-operatório * & FA-FA-FA \\
\hline 22 & Pré-operatório * & FA-FA-SA \\
\hline 23 & Pré-operatório * & FA-SA-FA \\
\hline 24 & Pré-operatório * & FA-FA-SA \\
\hline 25 & Pré-operatório * & FA-FA-SA \\
\hline 26 & Pré-operatório * & FA-FA-FA \\
\hline
\end{tabular}

* Os últimos 7 pacientes (20-26) possuíam apenas eletrocardiogramas realizados imediatamente antes da operação ou após a mesma.

** Na coluna "Evolução do ritmo", o primeiro ritmo (esquerda) é o pré-operatório, o segundo (meio) é o ritmo pós-operatório intra-hospitalar e o terceiro (direita), o ritmo pós-operatório tardio. Estão destacados os 10 pacientes em que houve reversão da FA para ritmo SA.

pós-operatório; naqueles com mais de 50 anos, este valor atingiu 60,0\%.

Os valores das variáveis ecocardiográficas estudadas no período pós-operatório são demonstrados na Tabela 5.

A mortalidade obtida foi de $3(5,7 \%)$ pacientes. Um (1,9\%) paciente faleceu tardiamente devido a endocardite bacteriana com embolia séptica para sistema nervoso central. Os outros $2(3,8 \%)$ morreram ainda no período intra-hospitalar por endocardite bacteriana com embolia séptica para sistema nervoso central e síndrome de baixo débito cardíaco por insuficiência mitral aguda, respectivamente.

\section{COMENTÁRIOS}

A freqüência de reversão de FA para ritmo sinusal obtida $(18,9 \%$ em relação ao total de pacientes; $38,5 \%$ em relação àqueles com $F A$ ) é maior se comparada ao valor obtido por HORSTKOTTE (5), 
Schmidlin C A, Loures D R R, Carvalho R G, Mulinari L A, Silva Jr. A Z, Brommelströet M, Choma R J, Shibata S, Leitão L A, Silva F R, Ultramari F T - Efeitos da correção cirúrgica de estenose mitral sobre o ritmo cardíaco. Rev Bras Cir Cardiovasc 2001; 16(2): 128-35.

\section{TABELA 4}

\begin{tabular}{|c|c|c|}
\hline \multicolumn{3}{|c|}{ COMPARAÇÃO DAS VARIÁVEIS ENTRE OS DOIS GRUPOS NO PERÍODO PRÉ-OPERATÓRIO } \\
\hline VARIÁVEL & GRUPO I & GRUPO II \\
\hline Idade em anos & $38,5 \pm 12,2$ & $50,0 \pm 10,6$ \\
\hline Faixa etária (\%) & $\begin{array}{l}1 \mathrm{a}: 4(11,4) \\
2^{\mathrm{a}}: 12(34,3) \\
3^{\mathrm{a}}: 9(25,7) \\
4^{\mathrm{a}}: 8(22,9) \\
5^{\mathrm{a}}: 1(2,9) \\
6^{\mathrm{a}}: 1(2,9) \\
7^{\mathrm{a}}: 0\end{array}$ & $\begin{array}{l}\left.1^{a}: 0\right) \\
2^{a}: 1(5,6) \\
3^{a}: 6(33,3) \\
4^{a}: 7(38,9) \\
5^{a}: 3(16,7) \\
6^{a}: 0 \\
7^{a}: 1(5,6)\end{array}$ \\
\hline № de operações da valva mitral (\%) & $\begin{array}{l}1: 23(65,7) \\
2: 10(28,6) \\
3: 2(5,7)\end{array}$ & $\begin{array}{l}1: 7(38,9) \\
2: 10(55,6) \\
3: 1(5,6)\end{array}$ \\
\hline Classe funcional pré-operatória (\%) & $\begin{array}{l}\text { I: } 1(2,9) \\
\text { II: } 11(31,4) \\
\text { III: } 21(60,0) \\
\text { IV: } 2(5,7)\end{array}$ & $\begin{array}{l}\text { I: } 1(5,6) \\
\text { II: } 1(5,6) \\
\text { III: } 15(83,3) \\
\text { IV: } 1(5,6)\end{array}$ \\
\hline Grau de estenose mitral (\%) & $\begin{array}{l}\text { Severa: } 27(77,1) \\
\text { Moderada: } 8(22,9)\end{array}$ & $\begin{array}{l}\text { Severa: } 11(61,1) \\
\text { Moderada: } 7(38,9)\end{array}$ \\
\hline Estenose mitral pura ou predominante (\%) & $\begin{array}{l}\text { Pura: } 16(45,7) \\
\text { Predominante: } 19(54,3)\end{array}$ & $\begin{array}{l}\text { Pura: } 5(27,8) \\
\text { Predominante: } 13(72,2)\end{array}$ \\
\hline Calcificação da valva mitral (\%) & $\begin{array}{l}\text { Sim: } 11(31,4) \\
\text { Não: } 24(68,6)\end{array}$ & $\begin{array}{l}\text { Sim: } 10(55,6) \\
\text { Não: } 8(44,4)\end{array}$ \\
\hline Átrio esquerdo em mm (\%) & $\begin{array}{l}\text { Aumento severo: } 10(28,6) \\
\text { Aumentado: } 25(71,4)\end{array}$ & $\begin{array}{l}\text { Aumento severo: } 7(38,9) \\
\text { Aumentado: } 11(61,1)\end{array}$ \\
\hline Gradiente transmitral médio $(\mathrm{mmHg})$ & $11,6 \pm 4,9$ & $12,4 \pm 5,1$ \\
\hline $\begin{array}{l}\text { Aumento da pressão sistólica da artéria } \\
\text { pulmonar em } \mathrm{mmHg}(\%)^{*}\end{array}$ & $\begin{array}{l}\text { Severo: } 14(53,8) \\
\text { Moderado: } 12(46,2) \\
\text { Normal: } 0\end{array}$ & $\begin{array}{l}\text { Severo: } 7(43,8) \\
\text { Moderado: } 8(50,0) \\
\text { Normal: } 1(6,3)\end{array}$ \\
\hline Fração de ejeção em \% (\%) & $\begin{array}{l}\text { Diminuída: } 20(55,9) \\
\text { Normal: } 15(44,1)\end{array}$ & $\begin{array}{l}\text { Diminuída: } 11(61,1) \\
\text { Normal: } 7(38,9)\end{array}$ \\
\hline Septo interventricular em mm (\%) & $\begin{array}{l}\text { Aumentado: } 3(8,6) \\
\text { Normal: } 32(91,4)\end{array}$ & $\begin{array}{l}\text { Aumentado: } 5(27,8) \\
\text { Normal: } 13(72,2)\end{array}$ \\
\hline Tipo de operação (\%) & $\begin{array}{l}\text { Comissur.: } 9(25,7) \\
\text { Comis+valvoplastia: } 4(11,4) \\
\text { Prótese biológica: } 12(34,3) \\
\text { Prótese metálica: } 10(28,6)\end{array}$ & $\begin{array}{l}\text { Comissur.: } 4(22,2) \\
\text { Comis+ valvoplastia: } 1(5,6) \\
\text { Prótese biológica: } 10(55,6) \\
\text { Prótese metálica: } 3(16,7)\end{array}$ \\
\hline
\end{tabular}

* Não foi possível verificar a pressão sistólica da artéria pulmonar em todos os pacientes (74,3\% dos pacientes do grupo l e $88,9 \%$ dos pacientes do grupo II), pois nem todos apresentavam refluxo tricúspide.

TABELA 5

VARIÁVEIS ECOCARDIOGRÁFICAS NO PERÍODO PÓS-OPERATÓRIO (6 MESES)

Área mitral média $\left(\mathrm{cm}^{2}\right)$

Átrio esquerdo médio $(\mathrm{mm})$

Gradiente transmitral médio $(\mathrm{mmHg})$

Pressão sistólica da artéria pulmonar média $(\mathrm{mmHg})$

Fração de ejeção média em \%

Septo interventricular médio $(\mathrm{mm})$
$2,4 \pm 0,7$

$49,1 \pm 10,4$

$5,8 \pm 4,6$

$46,8 \pm 14,8$

$63,3 \pm 9,0$

$9,3 \pm 1,7$ 
Schmidlin C A, Loures D R R, Carvalho R G, Mulinari L A, Silva Jr. A Z, Brommelströet M, Choma R J, Shibata S, Leitão L A, Silva F R, Ultramari F T - Efeitos da correção cirúrgica de estenose mitral sobre o ritmo cardíaco. Rev Bras Cir Cardiovasc 2001; 16(2): 128-35.

cujo estudo demonstrou a restauração do ritmo SA em $8 \%$ de 842 pacientes submetidos a correção cirúrgica da EM. Esses dados sugerem que a simples intervenção cirúrgica sobre a valva mitral não é suficiente para que ocorra reversão da FA para ritmo SA em uma parcela satisfatória dos casos. Em uma pesquisa com 189 pacientes submetidos a tratamento cirúrgico de EM, VOGT et al. (12) obtiveram conclusão semelhante: recomendam que os pacientes com elevado risco de desenvolver FA pós-operatória, isto é, idosos com história de FA prévia, tratamento anti-arrítmico prévio ou uma elevada pressão arterial pulmonar, devem ser considerados a reconstrução valvar e a um procedimento para FA combinados, mesmo se apresentarem ritmo SA no período pré-operatório.

A freqüência de degeneração do ritmo sinusal para FA obtida $(3,8 \%$ em relação ao total de pacientes; $7,4 \%$ em relação àqueles em ritmo $\mathrm{SA}$ ) é menor em relação à incidência de FA pós-operatória obtida após operações valvares em geral, nas quais pode atingir $36,7 \%(13)$.

A única variável que apresentou diferença estatisticamente significativa entre os grupos foi a idade. O estudo de CRESWELL et al. (14) com 297 indivíduos operados para correção de valvopatia, também encontrou, como único fator predisponente ao surgimento de FA após a operação, a idade. O risco de desenvolvimento de FA no período pósoperatório é diretamente proporcional ao aumento da idade. Há um risco relativo de 1,5 vez por década para o surgimento dessa arritmia em pacientes submetidos a operações valvares (13). As explicações para esse fenômeno são especulativas e incluem mudanças eletrofisiológicas relacionadas a alterações isquêmicas e atróficas do átrio.

Em outros estudos, diferentes variáveis foram confirmadas como fatores de risco para a FA pósoperatória. ASHER et al. (13), em uma pesquisa que incluiu 915 pacientes submetidos a operação valvar isolada, identificaram 4 fatores pré- e intra-operatórios: idade, átrio esquerdo aumentado, EM e reoperação como predisponentes à ocorrência desta arritmia e um fator protetor: hipotermia sistêmica. A possível contribuição da reoperação ao aumento de incidência de FA pós-operatória pode ser, na verdade, efeito do envelhecimento e dos progressivos efeitos hemodinâmicos e estruturais da valvopatia sobre o átrio esquerdo. O benefício da hipotermia sistêmica, reduzindo a taxa de pacientes que desenvolvem FA após a operação, também não foi comprovado e carece, como a influência da reoperação, de estudos confirmatórios.

A severidade do aumento atrial correlaciona-se com o risco aumentado de FA pós-operatória. En- tretanto, é importante ressaltar que mesmo em alguns pacientes com um severo aumento do átrio esquerdo, ocorre reversão da FA para ritmo SA após a operação. No presente estudo, dos 10 pacientes que apresentaram essa mudança após a operação, 4 tinham átrio esquerdo maior que 60 milímetros.

Diversos estudos avaliaram essas e outras variáveis e muitos resultados contraditórios foram observados entre eles. A área reduzida da valva mitral e o aumento da pressão do átrio direito foram os fatores mais intimamente associados à $F A$ em pacientes com EM, segundo MOREYRA et al. (15), cujo estudo não evidenciou uma relação significativa entre esta arritmia e a idade. UNVERFERTH et al. (16) não encontraram relação entre o desenvolvimento de FA e a pressão atrial esquerda, o gradiente transmitral e a área da valva mitral; mas sugeriram sua associação com a calcificação mitral. Segundo HORSTKOTTE ${ }^{(5)}$, 40\% dos pacientes com EM moderada estão com $\mathrm{FA}$, enquanto $93 \%$ daqueles com obstrução grave apresentam esta arritmia; além de evidenciar a importância da área da valva mitral, ressalta a idade como fator de risco para o surgimento da FA em pacientes com EM. Entretanto, o último autor relata uma fraca correlação entre FA e tamanho atrial esquerdo. De acordo com a análise de DIKER et al. (7), o diâmetro atrial esquerdo é o parâmetro mais importante que diferencia o paciente com e sem FA. Os três principais fatores predisponentes para desenvolvimento de FA em pacientes com EM pura ou predominante segundo ACAR et al. (4) são idade, diâmetro atrial esquerdo e calcificação mitral.

\section{CONCLUSÕES}

Vários estudos tentaram identificar os fatores predisponentes à permanência e ao desenvolvimento de FA após a operação, porém foram obtidos resultados contraditórios entre eles. No presente estudo, a única variável relacionada que apresentou associação com a FA foi a idade avançada. A operação de correção da EM apresenta resultados insatisfatórios em relação à reversão da FA para ritmo SA (apenas $38,5 \%$ ), sugerindo a necessidade de associação de outro procedimento cirúrgico para restaurar o ritmo normal. Apesar dos métodos profiláticos não estarem bem definidos, os pacientes com os prováveis fatores de risco para o surgimento da FA podem se beneficiar dos esforços preventivos ao desenvolvimento desta arritmia, como outros procedimentos cirúrgicos associados à correção da EM. 
Schmidlin C A, Loures D R R, Carvalho R G, Mulinari L A, Silva Jr. A Z, Brommelströet M, Choma R J, Shibata S, Leitão L A, Silva F R, Ultramari F T - Efeitos da correção cirúrgica de estenose mitral sobre o ritmo cardíaco. Rev Bras Cir Cardiovasc 2001; 16(2): 128-35.

RBCCV 44205-539

Schmidlin C A, Loures D R R, Carvalho R G, Mulinari L A, Silva Jr. A Z, Brommelströet M, Choma R J, Shibata S, Leitão L A, Silva F R, Ultramari F T - Effects of mitral stenosis correction surgery on the cardiac rhythm. Rev Bras Cir Cardiovasc 2001; 16(2): 128-35.

ABSTRACT: Objective: To determine the frequency of reversion of atrial fibrillation (AF) to sinus rhythm (SR) following surgical treatment of patients with mitral stenosis (MS) and identify likely favourable or unfavourable factors to this event.

Material and Methods: Retrospective case control study, analysing 53 patients with MS, without other valvar diseases, undergoing surgical correction. The baseline characteristics of the studied population were: women: 71.7\%; mean age: 42.4 years; functional class III: $67.9 \%$; mean mitral valve area: 0.92 $\mathrm{cm}^{2}$; mean left atrial size: $56.0 \mathrm{~mm}$; rhythm before the surgery: SR: $51.0 \%$ and AF: $49.0 \%$. Patients were divided in two groups according to their late postoperative rhythm: group I included the patients with SR after the surgery and group II were formed by those with AF in the postoperative period.

Results: Ten (18.9\%) patients out of all patients; $38.5 \%$ in respect to those with AF presented reversion of $\mathrm{AF}$ to $\mathrm{SR}$ and 2 of them (3.8\% out off all patients; $7.4 \%$ out of those with $\mathrm{SR}$ ) presented degeneration of rhythm from SR to AF. There was statistically significant difference $(p<0.05)$ between the groups only in respect to variable age $(p=0.0456)$.

Conclusions: The MS correction surgery presents unsatisfactory results in respect to reversion from AF to SR, suggesting the necessity of another associated surgical proceeding to restore the SR. Many studies tried to identify the predisposing factors to the permanence or to the development of AF after the surgery, but they got contradictory results. In the present study, only the variable advanced age presented association with AF.

DESCRIPTORS: Mitral valve stenosis, surgery. Atrial fibrillation, physiopathology. Heart rate, physiology.

\section{REFERÊNCIAS BIBLIOGRÁFICAS}

1 Kannel W B, Abbott R D, Savage D D, McNamara P M - Epidemiologic features of chronic atrial fibrillation: the Framingham study. N Engl J Med 1982; 306: 1018-22.

2 Kalman J M \& Tonkin A M - Atrial fibrillation: epidemiology and the risk and prevention of stroke. Pacing Clin Electrophysiol 1992; 15: 1332-46.

3 Melo J Q, Neves J P, Abecasis L M, Adragão P, Ribeiras $R$, Seabra-Gomes $R$ - Operative risks of the maze procedure associated with mitral valve surgery. Cardiovasc Surg 1997; 5: 112-6.

4 Acar J, Michel P L, Cormier B, Vahanian A, lung B Features of patients with severe mitral stenosis with respect to atrial rhythm: atrial fibrillation in predominant and tight mitral stenosis. Acta Cardiol 1992; 47: 115-24.

5 Horstkotte D - Arrhythmias in the natural history of mitral stenosis. Acta Cardiol 1992; 47: 105-13.

6 Ramsdale D R, Arumugam N, Singh S S, Pearson J, Charles R G - Holter monitoring in patients with mitral stenosis and sinus rhythm. Eur Heart $J 1987$; 8: $164-70$.

7 Diker E, Aydogdu S, Özdemir M et al. - Prevalence and predictors of atrial fibrillation in rheumatic valvular heart disease. Am J Cardiol 1996; 77: 96-8.

8 Sherrid M V, Clarck R D, Cohn K - Echocardiografic analysis of left atrial size before and after operation in mitral valve disease. Am J Cardiol 1979; 43: 171-8.
9 Zipes D P - Specific arrhythmias: diagnosis and treatment. In: Braunwald E, ed. Heart disease: a textbook of cardiovascular medicine. Philadelphia: WB Saunders, 1992: 682.

10 Selzer A - Effects of atrial fibrillation upon the circulation in patients with mitral stenosis. Am Heart J 1960; 59: 518-26.

11 Bailey G W, Braniff B A, Hancock E W, Cohn K E Relation of left atrial pathology to atrial fibrillation in mitral valvular disease. Ann Intern Med 1968; 69: 13-20.

12 Vogt $P$ R, Brunner-LaRocca H P, Rist $M$ et al. Preoperative predictors of recurrent atrial fibrillation late after successful mitral valve reconstruction. Eur J Cardiothorac Surg 1998; 13: 619-24.

13 Asher C R, Miller D P, Grimm R A, Cosgrove D M, Chung M K - Analysis of risk factors for development of atrial fibrillation early after cardiac valvular surgery. Am J Cardiol 1998; 82: 892-5.

14 Creswell L L, Schuessler R B, Rosenbloom M, Cox J L - Hazards of postoperative atrial arrhythmias. Ann Thorac Surg 1993; 56: 539-49.

15 Moreyra A E, Wilson A C, Deac R et al. - Factors associated with atrial fibrillation in patients with mitral stenosis: a cardiac catheterization study. Am Heart $J$ 1998; 135: 138-45.

16 Unverferth D V, Fertel R H, Unverferth B J, Leier C V - Atrial fibrillation in mitral stenosis: histologic, hemodynamic and metabolic factors. Int $\mathrm{J}$ Cardiol 1984; 5: 143-54. 